\title{
Cortical column design: a link between the maps of preferred orientation and orientation tuning strength?
}

\author{
Florentin Wörgötter ', Ernst Niebur ${ }^{2}$ \\ 1 Institut für Physiologie, Ruhr-Universität Bochum, D-44780 Bochum, Germany \\ 2 Computation and Neural Systems Program, California Institute of Technology, Pasadena, CA 91125, USA
}

Received: 5 November 1992/Accepted in revised form: 5 March 1993

\begin{abstract}
We demonstrate that the map of the preferred orientations and the corresponding map of the orientation tuning strengths as measured with optical imaging are not independent, but that band-pass filtering of the preferred orientation map at each location yields a good approximation of the orientation tuning strength. Bandpass filtering is performed by convolving the map of orientation preference with its own autocorrelation function. We suggest an interpretation of the autocorrelation function of the preferred orientations as synaptic coupling function, i.e., synaptic strength as a function of intracortical distance between cortical cells. In developmental models it has been shown previously that a "Mexican hat"-shaped synaptic coupling function (with a shape similar to that of the autocorrelation function) can produce a realistical-looking pattern of preferred orientations. Since optical imaging performs surface averaging, we discuss the possibility that the connection between the two maps is a measurement artifact of optical imaging. Whether this is the case can only be decided by combining electrode penetrations with optical imaging techniques for which we suggest experiments. We present a model for the generation of both maps from a single computational concept. The model is based on inverse Fourier transform of rather simple twodimensional annulus-shaped spectra which will produce a column structure very similar to real data. Thus, our approach shows that the complex appearance of cortical orientation columns has a rather simple description in the Fourier domain. Our theoretical analysis explains why singularities in the cortex do not have vorticities other than $\pm 1 / 2$, a result which corresponds to recent experimental findings. This study combines the results from several modeling approaches with recently available optical imaging data to construct a model of both aspects (angle and strength) of the cortical orientation column system. This could alter ideas about cortical development if the link between the two maps can be established as a physiological result.
\end{abstract}

Correspondence to: F. Wörgötter

\section{Introduction}

It has been known for several decades that visual cortical cells prefer elongated stimuli and that each cell has its individual preferred orientation (Hubel and Wiesel 1962) and its own orientation tuning strength. In the following report we will define orientation preference as the orientation measured in degrees of the visual field of a bar or grating-like stimulus leading to the strongest response. Orientation tuning strength will be defined as the rate at which responses fall off with increased difference between stimulus and preferred orientations. Neurons with high or low tuning strength respond over a narrow or broad range of angles, respectively. Orientation preferences are not randomly distributed over the cortical surface; instead, their organization follows several principles:

1. Except for isolated points (singularities) and possibly lines (fractures) (see Blasdel and Salama 1986; Blasdel $1992 \mathrm{a}, \mathrm{b})$, the preferred orientations change gradually along the cortical surface so that adjacent cells tend to have similar preferred orientations (Hubel and Wiesel 1968, 1974; Albus 1975; Humphrey and Norton 1980).

2. All preferred orientations occur within an approximately constant interval called a "hypercolumn" (Hubel and Wiesel 1963, 1974).

3. Plotting the preferred orientations against their surface location leads to curved slab-like structures that contain similar preferred orientations (Albus 1975; Blasdel and Salama 1986; Grinvald et al. 1986; Swindale et al. 1987; Ts'o et al. 1990; Bonhoeffer and Grinvald 1991).

4. At relatively few points of this interlaced structure all orientations meet. Such points are called "singularities" (Swindale 1982; Blasdel and Salama 1986; Swindale et al. 1987; Baxter and Dow 1989; Bonhoeffer and Grinvald 1991), and the angle of orientation preference at a singularity is not well defined (Swindale 1982; Baxter and Dow 1989).

5. Possibly, the orientation tuning strength is low in a singularity (Braitenberg and Braitenberg 1979; Swindale 1982; Götz 1987, 1988; Baxter and Dow 1989, 
but see Bonhoeffer and Grinvald 1991). In monkey many singularities are associated with zones of high cytochrome oxidase reactivity (cytochrome oxidase blobs, Blasdel 1992a, b).

6. Singularities can be characterized by their "vorticity" (Elsdale and Wasoff 1976; Swindale 1982; Baxter and Dow 1989) which is a measure of how often all preferred orientations occur in the vicinity of a singularity.

We will call these attributes the structural features of an orientation column system, because they are most prominent in cortical organization. In this study we will consider only the above-mentioned structural features even though more features might exist.

Many developmental models have been designed that reproduce some or all of the structural features of cortical orientation columns. Most models utilize reinforcement mechanisms between adjacent cells, starting off with an orientation bias which is either introduced as biased afferent convergence or develops as a result of the probabilistic noise in the system (von der Malsburg 1973; Nass and Cooper 1975; Perez et al. 1975; Cooper et al. 1979; von der Malsburg and Cowan 1982; Swindale 1982; Linsker 1986a, b; Miller 1990). Related to this approach is work which shows that self-organizing feature maps (Kohonen 1990) generate column structures which share many properties with orientation (or ocular dominance) columns in visual cortex (Obermayer et al. 1990, 1991). Similar maps are obtained if one assumes that the connections between neurons satisfy some geometric constraints (Soodak 1987). Basically, the maps minimize the total length of connections between like neurons, with the exception of a few long-range connections (Durbin and Mitchison 1990). In addition, some theoretical considerations have been published that tried to link the structural features to basic geometric design principles that could underlie cortical column systems (Braitenberg and Braitenberg 1979; Braitenberg 1985; Götz 1987, 1988; Baxter and Dow 1989; Bauer and Dow 1991). These approaches emphasize the distribution and vorticity of the singularities and regard them as possible foci from which the organization of a column system could be initialized (Braitenberg and Braitenberg 1979). In these reports, the actual initialization process and the development is not specified (but see Götz 1988). In yet another approach, Rojer and Schwartz (1990) were able to obtain realistic-looking orientation column structures by bandpass filtering of white noise. From such a multitude of models the question arises of whether there is a minimal model which captures the structural features of the cortex, subsuming the results from previous studies.

While the spatial pattern of orientation preference has recently been characterized using optical imaging techniques and a rather complete picture seems to emerge (Albus 1975; Blasdel and Salama 1986; Grinvald et al. 1986; Swindale et al. 1987; Ts'o et al. 1990; Bonhoeffer and Grinvald 1991; Blasdel 1992a, b), the spatial distribution of the orientation tuning strength is still a matter of controversy. Optical imaging reveals a low orientation tuning strength in the vicinity of singularities and fractures (Blasdel and Salama 1986; Bonhoeffer and
Grinvald 1991; Blasdel 1992a, b), which is in accordance with predictions of developmental as well as structural models of cortical maps (Braitenberg and Braitenberg 1979; Swindale 1982; Götz 1987, 1988; Baxter and Dow 1989; Obermayer et al. 1991). However, low orientation tuning strength may also be the result of spatial averaging involved in optical imaging. Experiments linking electrode penetration data with optical imaging results could, in principle, resolve this issue. The results reported so far, however, are still controversial (Blasdel and Salama 1986).

In our paper, as in many developmental approaches, we will focus on intracortical mechanisms which could produce cortical maps. First, we will investigate in detail the relationship between orientation preference and tuning strength as revealed by optical imaging. We will show that both maps are correlated and can be transformed into one another by a rather simple mathematical procedure. These results will then be juxtaposed with the hypothesis that the tuning strength map is an artifact of the optical imaging method and that it arises by spatial averaging. We will, however, adopt the point of view that the measured tuning strength map does, in fact, reflect the distribution of orientation selectivity in cortex, and we will investigate the consequences of this assumption. We will show that the link between orientation preference and tuning strength permits description of the properties of both maps (preferred orientation and orientation strength) with a minimal set of parameters which probably also underlie most of the previously proposed models of cortical map formation and structure (von der Malsburg 1973; Nass and Cooper 1975; Perez et al. 1975; Cooper et al. 1979; von der Malsburg and Cowan 1982; Swindale 1982; Linsker 1986a, b; Soodak 1987; Durbin and Mitchison 1990; Miller 1990; Obermayer et al. 1990, 1991).

\section{Linking the orientation maps}

In the following two sections we will show that the map of the orientation tuning strength can be obtained by band-pass filtering of the preferred orientation map. We will show that a straightforward way to compute this is to use the autocorrelation function of the map of the preferred orientations. Figure 1 gives an outline of the way we will present the results. The autocorrelation function is most easily computed using Fourier notation. Therefore, we will define the maps and develop the link between them in Fourier space. A second reason which justifies this procedure is that the two complicated-looking maps have a single consistent description in Fourier space, as will be shown below.

\subsection{Establishing the link}

The orientation tuning of a cortical cell can be described by the tuning strength and the angle of preferred orientation. Because the angle of preferred orientation is approximately constant in every column perpendicular to the cortical surface, we disregard the third dimension and 


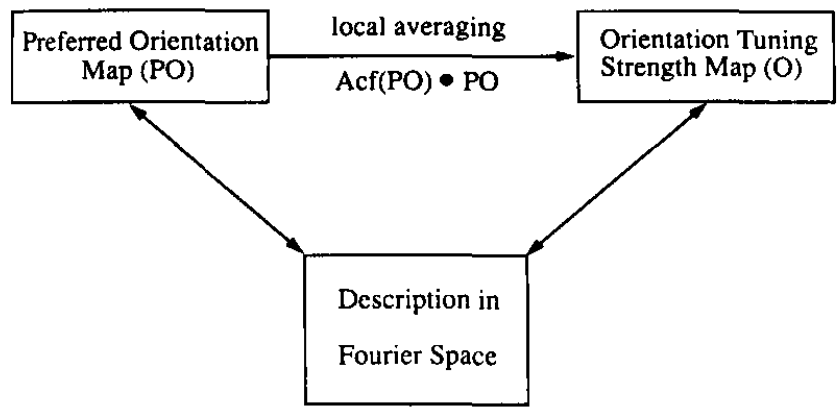

Fig. 1. Scheme for the computational process which transforms the map of the preferred orientations into the corresponding map of the orientation tuning strength

define the angle of the preferred orientation $\phi(x, y)$ and the orientation tuning strength $O(x, y)$ as functions of two variables $x$ and $y$ in the cortical plane. A concise notation is obtained by defining the preferred orientation in terms of a complex function $f(x, y)$ :

$$
\begin{aligned}
f(x, y) & =\operatorname{Re}[f(x, y)]+\mathrm{i} \operatorname{Im}[f(x, y)] \\
& =\cos [2 \phi(x, y)]+\mathrm{i} \sin [2 \phi(x, y)]
\end{aligned}
$$

where $\operatorname{Re}$ and $\mathrm{Im}$ describe the real and imaginary parts of the function. Following the method of Swindale et al. (1987), the angle of orientation preference $\phi(x, y)$ is then represented by*:

$\phi(x, y)=1 / 2 \arctan \frac{\operatorname{Im}[f(x, y)]}{\operatorname{Re}[f(x, y)]}$

Note that the amplitude

$\sqrt{\operatorname{Re}^{2}[f(x, y)]+\operatorname{Im}^{2}[f(x, y)]}$

is unity for all $(x, y)$. Let

$$
\begin{aligned}
F\left(k_{x}, k_{y}\right)= & \frac{1}{4 \pi^{2}} \iint f(x, y) \exp \left(-\mathrm{i} k_{x} x\right) \\
& \times \exp \left(-\mathrm{i} k_{y} y\right) \mathrm{d} x \mathrm{~d} y
\end{aligned}
$$

be the two-dimensional Fourier transform of $f(x, y)$. Then obviously we can write:

$\phi(x, y)=1 / 2 \arctan \frac{\operatorname{Im}\left\{\operatorname{IFT}\left[F\left(k_{x}, k_{y}\right)\right]\right\}}{\operatorname{Re}\left\{\operatorname{IFT}\left[F\left(k_{x}, k_{y}\right)\right]\right\}}$

where IFT denotes the inverse Fourier transform, defined as

$$
\begin{aligned}
\operatorname{IFT}\left[F\left(k_{x}, k_{y}\right)\right]=f(x, y)= & \iint F\left(k_{x}, k_{y}\right) \exp \left(\mathrm{i} k_{x} x\right) \\
& \times \exp \left(\mathrm{i} k_{y} y\right) \mathrm{d} k_{x} \mathrm{~d} k_{y}
\end{aligned}
$$

Although (5) is, of course, just a rewriting of the definition of $\phi$ in (2), it will help us to analyze the orientation columns in Fourier space. The usefulness of this approach is seen when we consider the power spectral

\footnotetext{
*Concerning angle doubling, the reader is referred to Batschelet (1981), Swindale (1982), and Swindale et al. (1987), who discuss at great length the resulting distortions of the spectra without angle doubling
}

density. Every Fourier spectrum $F\left(k_{x}, k_{y}\right)$ can be written as:

$$
\begin{aligned}
F\left(k_{x}, k_{y}\right) & =\left|F\left(k_{x}, k_{y}\right)\right| \exp \left[\mathrm{i} \Psi\left(k_{x}, k_{y}\right)\right] \\
& =\sqrt{P\left(k_{x}, k_{y}\right)} \exp \left[\mathrm{i} \Psi\left(k_{x}, k_{y}\right)\right]
\end{aligned}
$$

where $P\left(k_{x}, k_{y}\right)$ is the two-sided power spectral density and $\Psi\left(k_{x}, k_{y}\right)$, the phase spectrum.

Let us now consider the function

$$
\begin{aligned}
L\left(k_{x}, k_{y}\right) & =P\left(k_{x}, k_{y}\right) F\left(k_{x}, k_{y}\right) \\
& =F\left(k_{x}, k_{y}\right) F^{*}\left(k_{x}, k_{y}\right) F\left(k_{x}, k_{y}\right)
\end{aligned}
$$

where the asterisk denotes the complex conjugate. The amplitude of the inverse transformation of $L\left(k_{x}, k_{y}\right)$ is

$$
\begin{aligned}
& O(x, y) \\
& \quad=\sqrt{\operatorname{Re}^{2}\left\{\operatorname{IFT}\left[L\left(k_{x}, k_{y}\right)\right]\right\}+\operatorname{Im}^{2}\left\{\operatorname{IFT}\left[L\left(k_{x}, k_{y}\right)\right]\right\}}
\end{aligned}
$$

In the following paragraph we show that this quantity, which was derived from the map of the preferred orientations, is a description of the map of the orientation tuning strength from spectral information. Figure 1 summarizes the computational process.

Figure 2A, B shows cortical maps measured with optical imaging of intrinsic signals in cat area 17 (Bonhoeffer and Grinvald 1991). The map in Fig. 2A and all color maps which describe preferred orientations in the following figures are continuously color coded using a color circle for the maps of the preferred orientations and color coding from blue (weak tuning) to red (strong tuning) for the maps of the orientation tuning strengths (see figure legend for details). The similarity between measured and computed maps is shown by superimposing contours of equal tuning strength of the computed map (Fig. 2C) onto the measured map (Fig. 2B). Even finer features (e.g., small "bridges" between equal tuningstrength domains) match at most locations. In the computed map the differences between low and high tuning are more strongly pronounced than in the measured data, and most domains are somewhat broader. This leads to the overly high peak in the bottom left corner of the computed map (Fig. 2C) where the measured data show only a slight increase in tuning strength. The only severe mismatch occurs in the upper right corner where a narrow peak in the computed map does not find a clear correspondence in Fig. 2B. As an additional test we computed the euclidean distance between the two maps. First the maps $O_{1}$ (Fig. 2B) and $O_{2}$ (Fig. 2C) were normalized so that the lowest and highest values are represented by zero and one, respectively. Then the "distance" between the pixel values was computed as the square root of the normalized scalar product of the vectors given by the two images, i.e.,

$$
\operatorname{Dist}\left[O_{1}(x, y), O_{2}(x, y)\right]=\frac{1}{N} \sum_{(x, y)} \sqrt{\left[O_{1}(x, y)-O_{2}(x, y)\right]^{2}}
$$



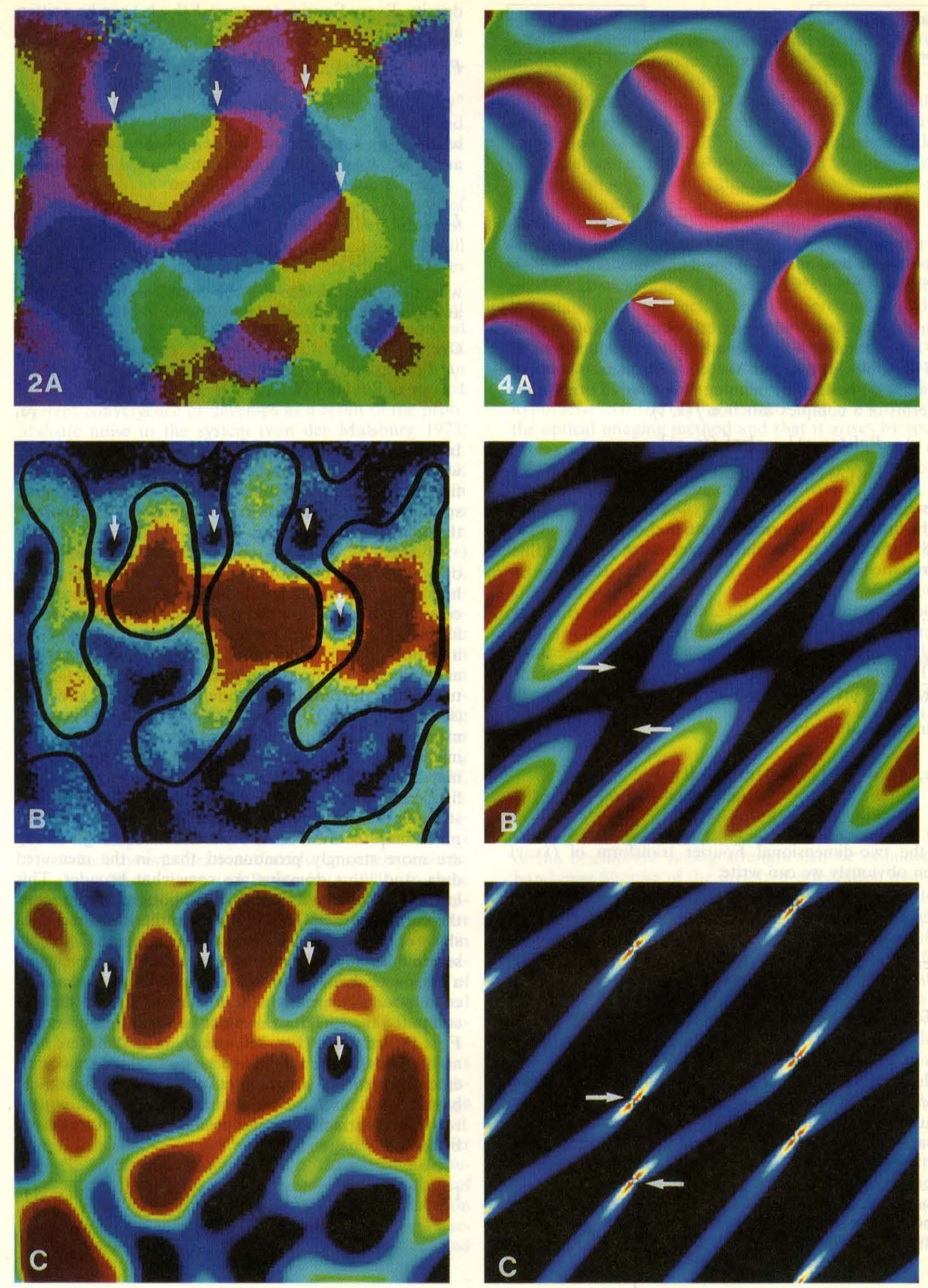
where $N$ is the total number of pixels in a map and the sum runs over all $(x, y)$ coordinates of the images. The chance distance is Dist $=0.5$. For the maps in Fig. 2 we found a value of 0.19 , which shows that the maps match closely at most locations. Quite similar results are obtained for other pairs of measured cortical maps.

\subsection{Analysis of the link between preferred orientation and orientation tuning strength}

As shown in the Appendix, the strength of the orientation tuning $O(x, y)$, given by $(9)$, is equivalent to band-pass filtering of the map of the preferred orientations, which is done by convolving the autocorrelation function of the preferred orientation map with this map itself. How can this be understood intuitively?

In previous reports it has been assumed that the orientation tuning map varies continuously for all cortical coordinates, with the possible exception of the "singularities" (Swindale 1982; Baxter and Dow 1989). At a singularity, all orientations "meet", i.e., cells with all preferred orientations can be found in the vicinity of the singular point. In order not to violate the assumption of continuity in a singularity, it follows (Baxter and Dow 1989; Obermayer et al. 1990) that the cells directly at the singularity must have vanishing orientation tuning strengths (i.e., they respond equally well to stimuli of all orientations). This is one possible way to explain the existence of spots with low orientation tuning strength at a singularity in the maps obtained with optical imaging.

Even if the assumption of continuity is dropped, however, the orientation tuning strength at a singularity is expected to be small if one averages the responses over some small neighborhood. This is clear because all orientations are found in the vicinity of the singularity. Averaging, therefore, generates equal responses to stimuli of all orientations, i.e., apparently vanishing orientation tuning strength. The crucial point is now that optical imaging methods always average over some area, whose size is given by the resolution of the method (for the data in Fig. 2 about 50-100 $\mu \mathrm{m}$; Frostig et al. 1990). As a consequence, in optical imaging, data singularities will always appear as regions of vanishing or very small orientation tuning strength, which is an inherent limitation of this technique.

We will concentrate now on the more interesting case that the link between the two cortical maps demonstrated before reflects a physiological situation. To achieve band-pass filtering, we computed (see Appendix) the convolution of the autocorrelation function with the preferred orientation map. It has been demonstrated in several reports that the autocorrelation function of the preferred orientation map is a monotonically decreasing positive function at small cortical distances and becomes negative with a much smaller amplitude at distances of about half a hypercolumn (Swindale et al. 1987; Obermayer et al. 1991). For distances larger than a hypercolumn width, the function is essentially zero. Thus, only values close to the regarded cortical coordinate will contribute significantly to the value of this convolution. In particular, after what we have said previously in this section, the convolution is zero or very small when close to a singularity because all preferred orientations coexist, and positive and negative contributions average out.

The question arises if simpler filtering kernels would give similar results. Low-pass filtering, which is equivalent to a local averaging of the map of the preferred orientations, appears as a possible alternative because this kernel is related to a Mexican hat by only omitting the negative peak. We have tried several gaussian-shaped kernels with different widths. For kernels with a width close to that of the first peak in the autocorrelation function, the results were similar to those mentioned before. The quality of the match between computed and measured map did not, however, exceed that obtained with the Mexican hat-shaped autocorrelation function kernel. In a gaussian kernel the width has to be manually adjusted, which is not necessary when using the autocorrelation function which represents a parameter-free model.

Therefore, three arguments support the procedure we used: (1) it allows for a closed description of both maps in the Fourier domain, (2) the autocorrelation function is a natural kernel for filtering because the kernel shape and width are implicitly defined by the preferred orientation map itself, and (3) there are reasons to believe that the autocorrelation function and the synaptic coupling function between cortical cells are related. We will elaborate on this last, most interesting, point in Sect. 6.

\section{A parsimonious model for orientation column structures}

In the previous section, we obtained a unified description of the map of preferred orientation as well as the map of the orientation tuning strength by transforming both to Fourier space. In this section, we will show that this technique is useful for generating pattern models which provide a very simple and parsimonious description of orientation columns. In addition, later sections will show that this parsimonious model together with some statistical fluctuations might actually reflect reality for cats, whereas for monkeys the model has to be further extended.

\footnotetext{
Fig. 2. Measured cortical maps of the preferred orientation $(\mathbf{A})$ and the orientation tuning strengths $(\mathbf{B})$ in cat and computed map of the orientation tuning strengths (C). Left, anterior; right, posterior; top, medial; bottom, lateral. A Color circle for the preferred orientations: $0^{\circ}$, dark blue; $22.5^{\circ}$, purple; $45^{\circ}$, red; $67.5^{\circ}$, orange; $90^{\circ}$, yellow; $112.5^{\circ}$, green; $135^{\circ}$, light blue; $157.5^{\circ}$, sky blue; $180^{\circ}$, dark blue. B, C Linear color scale for the orientation tuning strength (analog to a temperature scale); from weak to strong tuning, blue $\rightarrow$ green $\rightarrow$ yellow $\rightarrow$ red. Iso-orientation tuning strength domains from the computed map $(\mathbf{C})$ are plotted onto the measured map (B) to show the similarity of the maps. Arrows indicate singularities
}

Fig. 4. Preferred orientation map (A), orientation tuning strength map (B), and map of the degree of changes in the preferred orientations $(\mathbf{C})$ computed from a spectrum with three delta-function peaks at the same distance from the origin as shown in Fig. 3C. Arrows indicate two singularities where all colors meet. For color coding of $\mathbf{A}$ and $\mathbf{B}$ see legend of Fig. 2. Color coding of $\mathbf{C}$ : small change to large change, blue $\rightarrow$ red 
Swindale et al. (1987) computed the spectral density $\left|F\left(k_{x}, k_{y}\right)\right|$ of the cortical orientation column system. In our study we approximate the spectra they showed by three infinitely small peaks ("delta-function peaks") with constant distance from the origin at coordinates $\left(k_{x_{j}}, k_{y_{j}}\right)$, for $j=1,2,3, \ldots$ The phase component of the measured spectrum was not given in Swindale's report; we will set it to zero for all $\left(k_{x}, k_{y}\right)$. The Fourier spectrum $F\left(k_{x}, k_{y}\right)$ is then identical to the amplitude spectrum $\left|F\left(k_{x}, k_{y}\right)\right|$, or

$F\left(k_{x}, k_{y}\right)=\sum_{j=1}^{3} \delta\left(k_{x}-k_{x_{j}}, k_{y}-k_{y_{j}}\right)$

The inverse Fourier transformation can now be performed explicitly:

$$
\begin{aligned}
f(x, y) & =\int_{-\infty}^{\infty} \int_{-\infty}^{\infty} \exp \left(\mathrm{i} k_{x} x\right) \exp \left(\mathrm{i} k_{y} y\right) F\left(k_{x}, k_{y}\right) \mathrm{d} k_{x} \mathrm{~d} k_{y} \\
& =\sum_{j=1}^{3} \exp \left[\mathrm{i}\left(k_{x_{j}} x+k_{y_{j}} y\right)\right]
\end{aligned}
$$

Splitting into real and imaginary parts and applying (5), the preferred orientation is given by

$$
\phi(x, y)=1 / 2 \arctan \frac{\sum_{j=1}^{3} \sin \left(k_{x_{j}} x+k_{y_{j}} y\right)}{\sum_{j=1}^{3} \cos \left(k_{x_{j}} x+k_{y_{j}} y\right)}
$$

and the orientation tuning strength defined by (9) reads

$$
O(x, y)=\sqrt{\left[\sum_{j=1}^{3} \cos \left(k_{x_{j}} x+k_{y j} y\right)\right]^{2}+\left[\sum_{j=1}^{3} \sin \left(k_{x_{j}} x+k_{y_{j}} y\right)\right]^{2}} .
$$

Note that the function $L$ in (8) is defined (see appendix) as the inverse Fourier transform of the convolution of $f$ with the autocorrelation function of $f$. Equation 14 follows because in the singular case of $f$ being a combination of perfect sine waves [i.e., the Fourier transform being a sum of delta functions, (11)], $L$ will also be a sum of delta functions because the autocorrelation as well as the convolution of two sine functions remains a sine function of the same frequency.

A shift of the column structure with respect to the origin can be taken into account by including nonzero phases.

\subsection{Results from the simplified model}

To illustrate this simple model more clearly, we first describe (Fig. 3A, B) some examples of orientation column systems obtained from (13) that use fewer than three delta-function peaks in the spectrum.

With a single peak (Fig. 3A) located on the $k_{x}$ axis, (13) is reduced to

$\phi(x, y)=\frac{1}{2} \arctan \frac{\sin \left(k_{x_{1}} x\right)}{\cos \left(k_{x_{1}} x\right)}=\frac{1}{2} k_{x_{1}} x$.

The change of the orientation angle $\phi$ (Fig. 3B) is linear in $x$, and there is no change in $y$, so that a parallel straight

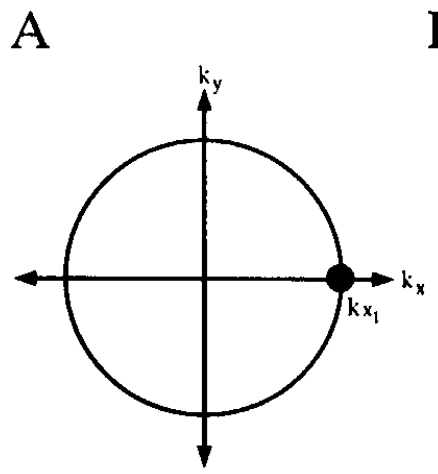

B
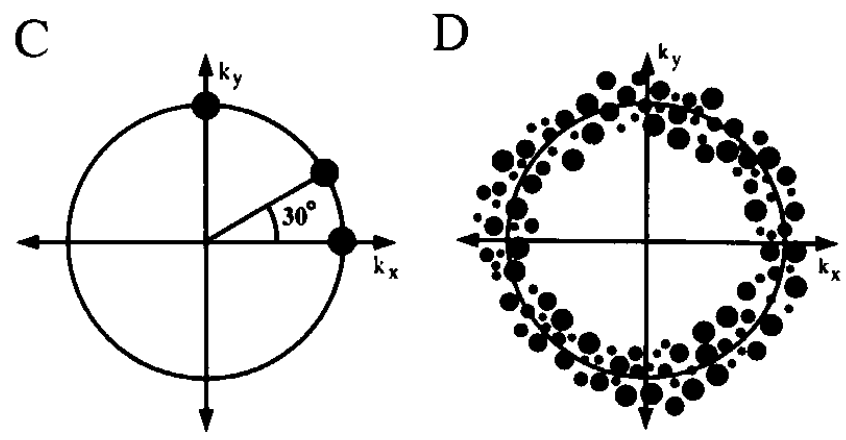

Fig. 3. A, B Schematic spectrum with only one delta-function peak located on the $x$-axis and corresponding column structure. C Spectrum with three delta-function peaks at the same distance from the origin arranged with angles $0^{\circ}, 30^{\circ}$, and $90^{\circ}$ with respect to the $x$-axis. This spectrum yields the maps shown in Fig. 4. D Annulus-like spectrum with multiple peaks of different amplitudes and approximately constant distance from the origin. This spectrum yields the maps shown in Fig. 6

column structure is obtained. Since $k_{y_{1}}$ is zero, $k_{x_{1}}$ is directly related to the wavelength $\lambda$ of the pattern $\lambda=\frac{2}{k_{x}}$. The wavelength is the hypercolumn width in this model. Note that the wavelength is given as twice the inverse of the distance from the DC component to the peak in the Fourier spectrum. Because the numerical computation of spectra from preferred orientation maps require an angle doubling as described previously, the frequencies are doubled in the spectrum, and the hypercolumn width (as the inverse of a frequency) has to be corrected by multiplying by 2 .

There is evidence that the hypercolumn width is approximately the same in all directions (Hubel and Wiesel 1974; Albus 1975; Obermayer et al. 1991); therefore, additional peaks must be located, all at about the same distance $\lambda^{-1}$ from the origin. It is easy to see that two peaks yield straight, parallel patterns which are tilted with respect to the coordinate axes.

Figure 4 shows an example of a column structure obtained from (13), i.e., with three delta-function peaks in the amplitude spectrum (Fig. 3C). The map of the preferred orientations (Fig. 4A) contains all six structural features of cortical orientation columns in cat which were defined in the Sect. 1. Singularities (two of which are marked by arrows in the figure) arise where all colors meet. At most points, however, the preferred orientations change gradually, i.e., singularities are rare. The gradient of the orientation change is small in most places, but the 
singularities are linked by stretches with a larger gradient (Fig. 4C), the so-called fractures (Blasdel and Salama 1986).

Figure 4B shows the color-coded maps of the orientation tuning strength (14) which correspond to the preferred orientation map as shown in Fig. 4A. Blue represents weak, red represents strong orientation tuning. The pattern shows a "blob" and "interblob" structure of alternating weak and strong orientation tuning. The orientation tuning disappears in the singularities, some of which are marked as in Fig. 4A.

\section{Real spectra}

The concept of a hypercolumn (Hubel and Wiesel 1974) assumes a modular organization of the cortex (Szentagothai 1975) and leads to a periodic repetition of the different cell characteristics, including orientation specificity. It can be shown (Bracewell, 1986, p $224 \mathrm{ff}$ ) that the repetitions cannot have exactly the same periodicity along every direction in the cortical surface, since this could only hold for a single "center" location and would lead to a cortex with preferred orientations spreading like circular waves from this center. What can be obtained is constant periodicity on average in all directions. The Fourier spectrum which corresponds to a column structure with this property is an annulus of a certain radius and width containing Fourier peaks of various amplitudes (Fig. 3D). The width of the annulus represents the variability of the frequency, and the average radius of the annulus represents the average periodicity (i.e., repetition frequency). The inverse of the average repetition frequency can then be defined as the width of a hypercolumn, as measured by Fourier analysis of a cortical orientation column system.

The spectrum of an experimentally obtained map of the preferred orientations in visual area $\mathrm{V} 1$ of monkey is shown in Fig. 5 (Obermayer et al. 1991). The annulus shape is quite obvious for the monkey data (Fig. 5) where $512 \times 512$ pixels could be used for Fourier analysis. The average hypercolumn width is $700 \mu \mathrm{m}$ as measured from the spectrum. In previous reports estimates of the hypercolumn width of $570 \mu \mathrm{m}$ (Hubel et al. 1978) or $640 \mu \mathrm{m}$ (Hubel and Wiesel 1974) were given by applying other methods.

Much lower resolution data $(128 \times 128)$ were available for cat which were not sufficient to yield a clear shape in the spectrum. Therefore, this spectrum is not shown here; instead we refer the reader to the work of Diao et al. (1990). In Fig. 8 of their paper, power spectra for cat data are shown that display a clear blob-like arrangement. Such an arrangement would be the direct consequence of our parsimonious model if the center frequencies would only be spread out to blobs of a certain width.

Thus, in the following discussion we restrict ourselves to monkey data. Figure $6 \mathrm{~A}-\mathrm{C}$ shows maps of the preferred orientations, the orientation tuning strengths, and the fractures, obtained by applying (9) and (5) to an artificial (and therefore ideal) annular Fourier spectrum

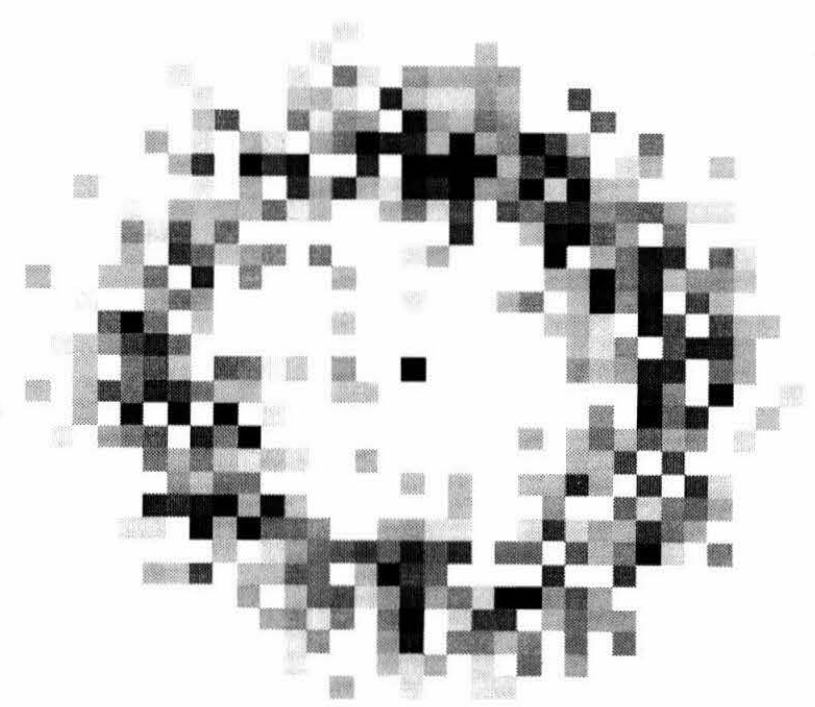

Fig. 5. Power spectrum from monkey orientation column map. Field size for the monkey data was $512 \times 512$ pixels and an annulus-shaped spectrum results. The hypercolumn with $\lambda$ equals $700 \mu \mathrm{m}=2$ /annulus radius. The factor of two is due to the angle doubling in (1). The strong DC component (in the center of the figure) is probably artificially introduced to a large degree as a border effect because the size of the full preferred orientation map divided by the hypercolumn width is not an integer

exemplified in Fig. 3D. Comparison with the data from Blasdel and Salama (1986) shows the similarity between these maps and the corresponding maps obtained from measured data. We will discuss possible differences between cat and monkey data in Sect. 5. Note that the gradient at a fracture is not very strong: between two singularities it is by a factor 10 smaller than close to a singularity. The line-like appearance of the fractures corresponds very well to the data of Blasdel and Salama (1986) and Bonhoeffer and Grinvald (1991).

\section{Singularities and vorticity}

As described in Sect. 1, there are many approaches which produce realistical looking orientation columns. Analysis by "looking at the results", however, is not necessarily a good criterion for distinguishing which of the models comes closest to a correct description of reality. Therefore, it is important to develop other criteria which allow us to distinguish between different models and allow for a comparison with the physiological maps. In this section, we will argue that there is probably only one type of singularity in the cortex, which also gives a criterion to judge how realistic a particular model map is.

Annulus spectra naturally lead to singularities in the pattern of orientation preferences. Singularities can be characterized by their "topological index" (Elsdale and Wasoff 1976), which is a measure of the vorticity at the singularity and indicates the amount of rotation of the angular field around the singular point. The topological index of a point is computed by integrating the differential orientation angle on a counterclockwise path which goes exactly once around the point (and does not include 

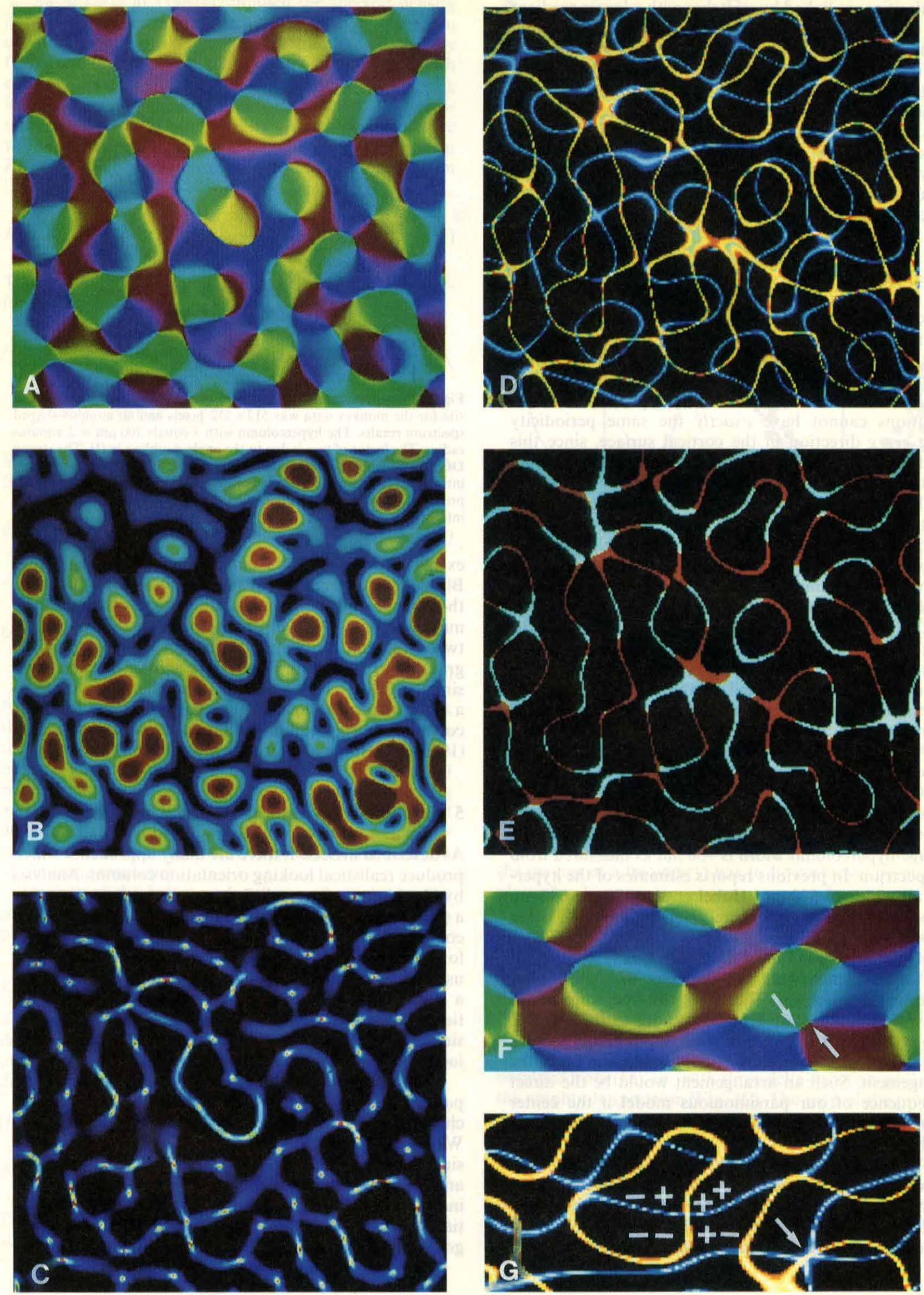
another singularity). It is customary to divide the result by $2 \pi$. At a singularity this integral will generally be different from zero. Twice the value of the topological index indicates the number of times all orientations occur in the vicinity of a singularity, and its sign indicates the direction of rotation of the preferred orientations (Swindale 1982; Baxter and Dow 1989; Bauer and Dow 1991).

\subsection{Vorticity in different models}

In the model proposed by Swindale (1982, 1985), the preferred orientation is given by the function $1 / 2$ $\arctan \frac{a(x, y)}{b(x, y)}$, and singularities occur at simultaneous zeros of both functions $a$ and $b$. From the annulus spectrum in Fig. 3D we computed the numerator $a(x, y)=\operatorname{Im}\left\{\operatorname{IFT}\left[F\left(k_{x}, k_{y}\right)\right]\right\}$ and the denominator $b(x, y)=\operatorname{Re}\left\{\operatorname{IFT}\left[F\left(k_{x}, k_{y}\right)\right]\right\}$ from (5) and plotted the line-shaped regions where both functions $a(x, y)$ (blue) and $b(x, y)$ (yellow) are close to zero (Fig. 6D). As in the mdel of Swindale et al., those functions appear to meet at the singularities (see below). The important point now is that the probability for more than two such lines to cross exactly at the same location is zero because the two functions we are considering are smooth and periodic (i.e., not constant). One such spot where two crossing points apparently fall together (crossing of four lines) is marked (arrows) in the magnified figures (Fig. 6F, G). A closer inspection (Fig. $6 \mathrm{~F}$ ) reveals, however, that at this location the crossing points are very close to each other, but that they do not coincide, and this region still contains two and not one singularity.

The singularity marked with sign symbols $( \pm)$ in the four quadrants (Fig. 6G) demonstrates that the vorticity at the crossing point is either $+1 / 2$ or $-1 / 2$. The signs refer to the numerator and denominator of the argument of the arctan function in (5) [or (2)], and in such a fourquadrant system each orientation occurs exactly once in the vicinity of a singularity. Singularities with absolute vorticity values larger than $1 / 2$ require crossing of more than two lines.

The conclusion at this point is that, provided our description complies with reality, all the singularities in the cortex are of vorticity $+1 / 2$ or $-1 / 2$.

That only the lowest possible vorticity values are implemented in the cortex can also be interpreted developmentally. Periodicity arises as a consequence of the multimodular (i.e., pixel) representation in the cortex. That there is only one predominant spatial frequency (and not more, e.g., a double annulus) is consistent with the concept that the properties of the neurons vary as smoothly as possible in the cortical plane. In general, a function is said to be "smooth" if it is continuous and has small derivatives. Thus, the smoothest periodic map- ping of a periodic variable (e.g., orientation) is obtained with a linear mapping function, which has a constant first derivative and vanishing higher derivatives. Linear variation of the preferred orientation (interupted by discontinuities due to singularities) is indeed observed generically along electrode tracks (Hubel and Wiesel 1974). Consequently, the lowest possible vorticity in a singularity also reflects the smoothest possible implementation of a cortical discontinuity.

Above we stated that in our model the functions $a$ and $b$ apparently cross. In our model it is obvious that simultaneous zeros of both numerator and denominator in (2) cannot exist, since this would violate (3). Following (3), the denominator must be +1 or -1 if the numerator is zero and vice versa. Thus, a singularity is a true discontinuity. Figure $6 \mathrm{E}$ shows the domains where the denominator is either close to +1 (white) or -1 (red). At the singularities a jump from -1 to +1 occurs. The functions describing the paths of the zeros in the numerator and denominator (e.g., Fig. 6D) can be continuously filled in at their crossings. The discontinuity is a "hole". The corresponding functions which follow the paths of +1 or -1 (e.g., Fig. $6 \mathrm{E}$ ) have jumps at the locations of the singularities which cannot be filled in continuously.

\section{Discussion and conclusion}

In this paper we have focused on intracortical mechanisms which could produce cortical maps. As in many developmental approaches, an existing afferent bias (Ferster 1987; Chapman et al. 1991) is only implicitly included as the possible starting point of the development of the maps. Despite a huge body of work on the development and structure of maps of preferred orientation, essentially nothing is known about the maps of the orientation tuning strength, for which data have become available only recently. In this report, we have therefore focused on this topic as well as on the relationship between the two maps. We will first compare our model to related approaches and summarize the basic findings of our study before we discuss the details of the results.

\subsection{Related models}

Our approach is related to the models of Swindale (1982, 1985, 1992) and of Rojer and Schwartz (1990). In the developmental model of Swindale (1982) the change of the preferred orientations between two developmental steps is determined by a Mexican hat-shaped coupling function. The actual strength of the change exerted from one region onto another region is given by a function of the modulus $|\mathbf{z}|=\sqrt{a^{2}+b^{2}}$, where $a$ and $b$ are the

\footnotetext{
Fig. 6A-G. Maps computed from the annulus-like spectrum shown in Fig. 3D. A Preferred orientation map. B Orientation tuning strength map. C Map of the degree of changes in the preferred orientations. Color coding as in Fig. 4. D Domains where the numerator (blue) and the denominator (yellow) of (5) are close to zero. The brighter (i.e., more white) the color, the closer is the value to zero. Singularities arise where both functions "cross". E Plot of the denominator in the domains where the numerator is close to zero. White, Denominator close to +1 ; red, denominator close to -1 . Note the jumps at the singularities. F, G Magnifications from $\mathbf{A}$ and $\mathbf{D}$. The arrows mark a singularity with apparent vorticity other than $\pm 1 / 2$. Close inspection of $\mathbf{F}$ shows that this location actually contains two singularities very close to each other. Sign symbols give the signs of numerator and denominator of (5) close to a singularity
} 
components of a vector $\mathbf{z}$ and the preferred orientation is defined as $\phi=\frac{1}{2} \arctan \frac{\theta}{b}$. In his reports of 1982 and 1985 , Swindale associates the modulus $|\mathbf{z}|$ with the orientation tuning strength. He alluded to this concept in 1992, but did not give a description of the actual shape of the link between the orientation maps; instead this study strongly focused on the relationship between ocular dominance and orientation preference. To a certain degree our approach appears similar to that of Swindale. Swindale, however, describes a developmental model which generates the preferred orientation map iteratively. Our approach, on the other hand, focuses on the final state of the system, and we tried to give a simple analytical description of the preferred orientation map as well as of the map of the orientation tuning strengths. The shapes of the "coupling" functions in Swindale's and our approach are similar (i.e., Mexican hat). We extended his concept and suggested in our study that the (Mexican hat-shaped) autocorrelation function of the preferred orientation map might directly reflect the synaptic coupling onto a given cell. Note that this assumption only deals with the net excitatory or inhibitory effect as a function of distance; therefore, we cannot draw conclusions about subliminal excitation or inhibition occurring simultaneously with their much stronger, and thus dominating, antagonistic counterparts. The autocorrelation function appears to be a natural choice for the Mexican-hat functions that appear in Swindale's and other reports (von der Malsburg 1973; Nass and Cooper 1975; Legendy 1978; Swindale 1982; Linsker 1986a, b; Miller 1990) because the autocorrelation function inherently represents local correlation and medium distance anticorrelation.

In 1987, Swindale et al. used Fourier methods to analyze cortical column structures. At that time only the first reports had appeared on optical imaging of the visual cortex (Blasdel and Salama 1986; Grinvald et al. 1986). We were able to analyze optical imaging data and confirm that a "modulus" as in (9) can actually reflect the orientation tuning strength. The definition of the modulus, however, is somewhat more complicated than that proposed by Swindale because the functions $a$ and $b$ from his definition of the preferred orientation cannot be used directly. Singularities occur in Swindale's (1985) model and in our model at the joint zero-crossings of the functions $a$ and $b$. At that time no final conclusion could be drawn about the actual vorticity of the singularities. In our model we were able to give theoretical reasons that the vorticity of the singularities in cortical maps is probably always $\pm 1 / 2$. This is strongly supported by the most recent data (Bonhoeffer and Grinvald 1991).

Rojer and Schwartz (1990) explicitly use an annulus spectrum to generate the map of the preferred orientations in monkey by a computational process very similar to the one we used, but they restricted their analysis to the case of orientation preference only. We have extended this analysis to include orientation tuning strength and provided a closed description of both maps. Rojer and Schwartz (1990) proposed that a completely isotropic annulus spectrum will better fit monkey than cat orientation column data. We agree with them on this point, but do not wish to elaborate on it; instead, we refer the reader to their detailed study.

\subsection{Relation between preferred orientation and orientation tuning strength}

Orientation tuning strength varies with the stimulus parameters used. It can change with the shape of the stimulus (e.g., grating vs bar), the velocity, spatial frequency, and other parameters. The preferred orientation is much less affected by those variations. In view of this, the following discussion will treat the map of the orientation tuning strength as a relative measure dependent on the particular stimulus used. It can be assumed, however, that a change in the stimulus parameters will affect all regions of the map in the same way. It is likely that regions with different orientation tuning strength will maintain this difference (perhaps on a changing scale) even though the absolute tuning strength value is shifted up or down by a change in stimulus.

As was already mentioned, current optical imaging methods always average over an area of a diameter not smaller than $50 \mu \mathrm{m}$ and often larger. In the light of this limitation, we have to ask if the observed relation between the maps of preferred orientation and orientation tuning strength is an artifact of the optical imaging method. This question has to be decided by electrode experiments which have a much higher resolution.

The orientation tuning strength in one column seems to be fairly constant over depth ranges of several hundreds of micrometers (Lee et al. 1977) but can change significantly between the cortical layers [Orban (1984) reviews these results]. Since optical imaging also performs depth averaging over 100-300 $\mu \mathrm{m}$ (Bonhoeffer and Grinvald 1991), tuning constancy over depth is a prerequisite without which the corresponding map would only reflect an averaging effect.

Based on the assumption that the column system is continuous, several authors have suspected that singularities coincide with regions of low orientation tuning, which would mean that there is a physiological link between the maps of preferred orientation and the orientation tuning strength. There is some evidence for this in monkey. Here singularities seem to be associated with cytochrome oxidase blobs which contain predominantly cells with low orientation tuning (Blasdel 1992a, b). Bonhoeffer and Grinvald (1991 and personal communication) reported that they found mostly cells with significant orientation tuning strength when recording with an electrode in a singularity. Edwards and Kaplan (1992), on the other hand, reported that they observed both situations close to a singularity: either predominantly cells with low orientation tuning or simply a high degree of orientation scatter with regularly tuned cells. Thus, at this point no clear conclusions can be drawn concerning this question.

Another argument in favor of a physiologically relevant link is based on present theories of cortical development. Very likely, intracortical interaction plays an important role in the development of the orientation column system. Most developmental models assume 
synaptic links which reinforce the similarity between the orientation preferences of adjacent cells (positive correlation). The strength of this reinforcement mechanism decreases with distance, and most models introduce inhibitory coupling at larger distances, which results in a negative correlation of the activity of the cells which then develop (nearly) orthogonal orientation preferences (von der Malsburg 1973; Nass and Cooper 1975; Legendy 1978; Swindale 1982; Linsker 1986a, b; Miller 1990, 1992).

There is physiological evidence that such a Mexican hat-shaped coupling function is reflected in the distribution and weighting of the synapses onto a given cell (Hess et al. 1975; Toyama et al. 1981a, b; Hata et al. 1988; Wörgötter and Eysel 1991). As previously explained, a Mexican hat-shaped coupling function will lead to a similarly shaped autocorrelation function for the preferred orientations. Thus, the shape of the autocorrelation function might directly mirror the shape of the synaptic input function with central excitation and peripheral circularly arranged inhibition (Niebur and Wörgötter 1990; Wörgötter et al. 1991) at a distance of half a hypercolumn, which amounts to a weakly tuned "cross-orientation inhibition".

As a consequence of the shape of the synaptic coupling function, the synaptic input for any stimulus orientation is closely related to the preferred orientations in the cortical environment of the cell. The input would be strongly orientationally tuned for neurons surrounded by cells with the same preferred orientations. Untuned or weakly tuned input would arise if adjacent cells had widely varying orientation preferences. Thus, convolving the synaptic coupling function $(\approx$ autocorrelation function) with the actual pattern of preferred orientations should give a measure of the orientation tuning strength of the input at every location (see appendix). The expected consequence is that a strongly tuned input leads to strong orientation tuning in the target cells itself, and vice versa.

\subsection{Simplicity of orientation columns}

The representation of the filtering process by the correlation function in Fourier space yielded a unified description of the maps of preferred orientation and orientation tuning strength. In addition, the Fourier formalism provided a series of simple models which generate surprisingly "good-looking" orientation column systems. Our models are not supposed to give any detailed insight into developmental mechanisms; instead, their virtue is that they are among the most parsimonious models proposed for the generation of orientation columns. Our simplest model has three peaks in Fourier space, and the Fourier transformation can be done analytically. The model can thus be expressed in the form of analytical expressions, which makes it particularly attractive for the generation of simple but realistic column structures for use as a component in other models or in computer simulations (Niebur and Wörgötter 1990; Wörgötter et al. 1990, 1991). Our most realistic model is completely specified by an annulus of intensities in Fourier space, i.e., by the annulus diameter and thickness. We have shown that this model is capable of producing orientation column maps whose realism rivals that of the most sophisticated developmental models, at least if one judges by the visual appearance of the maps produced. Therefore, it seems that very simple and generic models are sufficient to explain the apparently complicated orientation column structure if an appropriate representation is chosen. In addition to the simplicity of this approach its power lies also in the fact that a combination of such a model with an isotropic connection scheme ("circular inhibition") leads to nontrivial features, such as direction and orientation selectivity, which was quite unexpected (Wörgötter et al. 1991; Wörgötter and Niebur 1992).

\subsection{Suggested experiments}

It seems appropriate to conclude our theoretical paper with suggestions for experimental work. We propose to investigate the question whether the described relation between the map of the orientation tuning strength and the map of the orientation tuning is an artifact of the optical imaging method or a physiological effect. At this moment, no clear decision can be made between the two interpretations, but several experiments could lead to a conclusion. The first test would be a quantitative analysis of the change in orientation tuning strength with recording depth in a single column. Depth constancy is a requirement without which a physiological link in the proposed form does not seem possible. Provided this holds, the second test could assess the change of orientation tuning strength between closely spaced penetrations which should be smooth if a link between the maps exists. The crucial final test would involve a quantitative analysis of the tuning strength obtained with microelectrodes at different locations in a previously measured map of the preferred orientations. It should be low if the preferred orientations change fast.

Acknowledgements. We thank Drs. T. Bonhoeffer, A. Grinvald, and G. Blasdel for sharing their data with us. Discussions with Ken Miller and Klaus Obermayer are gratefully acknowledged. E.N. is supported by the Air Force Office of Scientific Research and the National Science Foundation (NSF), and F.W. by the Deutsche Forschungsgemeinschaft. The authors gratefully acknowledge the support by the Air Force Office of Scientific Research for an NSF Presidential Young Investigator Award and the James S. McDonnell Foundation to Christof Koch. We would like to thank Christ of Koch and Ulf Eysel for their ongoing encouragement and support.

\section{Appendix: orientation tuning, convolution, and autocorrelation functions}

We show here that the orientation tuning strength as defined in (9) is essentially identical to the convolution of the map of preferred orientations with its own autocorrelation function. We will perform the derivation in one dimension, where equations are simpler than in two dimensions; everything is, however, completely analogous in two dimensions. 
From the one-dimensional version of (4), and after omitting factors of $2 \pi$, the Fourier transform of $f$ is found as

$F(k)=\int \mathrm{e}^{\mathrm{i} k x} f(x) \mathrm{d} x$

and its complex conjugate is

$F^{*}(k)=\int \mathrm{e}^{\mathrm{i} k x} f^{*}(x) \mathrm{d} x$.

As a consequence, the one-dimensional version of $(8)$ is

$$
\begin{aligned}
L(k) & =F^{*}(k) F(k) F(k) \\
& =\int \mathrm{e}^{\mathrm{i} k y} f^{*}(y) \mathrm{d} y \int \mathrm{e}^{-\mathrm{i} k u} f(u) \mathrm{d} u \int \mathrm{e}^{-\mathrm{i} k v} f(v) \mathrm{d} v
\end{aligned}
$$

where we have renamed the integration variables. Collecting terms, we get

$$
\begin{aligned}
F^{*}(k) F(k) F(k) & =\iiint \mathrm{e}^{\mathrm{i} k(y-u-b)} f^{*}(y) f(u) f(v) \mathrm{d} u \mathrm{~d} y \mathrm{~d} v \\
& =\int \mathrm{e}^{-\mathrm{i} k x} \mathrm{~d} x \int f(u) \mathrm{d} u \int f(v) f^{*}(v-x+u) \mathrm{d} v
\end{aligned}
$$

This is the inverse Fourier transformation of the function

$$
\int f(u) \mathrm{d} u \int f(v) f^{*}(v-x+u) \mathrm{d} v .
$$

Now, from the definition of the complex autocorrelation function of $f(v)$ (see Bracewell 1986),

$f \star f(x)=\int f(v) f^{*}(v-x) \mathrm{d} v$

and of the convolution of two functions, $f$ and $g$,

$f \circ g(x)=\int f(u) g(x-u) \mathrm{d} u$

it is clear that (21) represents the convolution of $f(x)$ with its own autocorrelation function, i.e.,

$F^{*}(k) F(k) F(k)=\int \mathrm{e}^{-\mathrm{i} k x} f \circ f \star f(x) \mathrm{d} x$.

We have thus shown that inverse Fourier transformation of $F^{*}(k) F(k) F(k)$ yields the convolution of $f(x)$ with its own autocorrelation function.

\section{References}

Albus K (1975) A quantitative study of the projection area of the central and the paracentral visual field in area 17 of the cat. II. The spatial organization of the orientation domain. Exp Brain Res $24: 159-179$

Batschelet E (1981) Circular statistics in biology. Academic Press, New York

Bauer R, Dow BM (1991) Local and global principles of striate cortical organization: an advanced model. Biol Cybern 64:477-483

Baxter WT, Dow BM (1989) Horizontal organization of orientationsensitive cells in primate visual cortex. Biol Cybern 61:171-182

Blasdel GG (1992a) Differential imaging of ocular dominance and orientation selectivity in monkey striate cortex. J Neurosci 12:3115-3138

Blasdel GG (1992b) Orientation selectivity preference and continuity in monkey striate cortex. J Neurosci 12:3139-3161

Blasdel GG, Salama G (1986) Voltage-sensitive dyes reveal a modular organization in monkey striate cortex. Nature 321:579--585

Bonhoeffer T, Grinvald A (1991) Iso-orientation domains in cat visual cortex are arranged in pinwheel like patterns. Nature 353:429-431
Bracewell RN (1986) The Fourier transform and its applications. Academic Press, London

Braitenberg V (1985) Charting the visual cortex. In: Peters A, Jones EG (eds) Cerebral cortex, vol 3. Plenum, New York, pp. 379-414

Braitenberg V, Braitenberg C (1979) Geometry of orientation columns in the visual cortex. Biol Cybern 33:179-186

Chapman B, Zahs KR, Stryker MP (1991) Relation of cortical cell orientation selectivity to alignment of receptive fields of geniculocortical afferents that arborize within a single orientation column in ferret visual cortex. $\mathrm{J}$ Neurosci $11: 1347-1358$

Cooper LN, Fishel, Liberman, Oja E (1979) A theory for the acquisition and loss of neuron specificity in visual cortex. Biol Cybern 33:9-28

Diao YC, Jia WG, Swindale NV, Cynader MS (1990) Functional organization of the cortical $17 / 18$ border region. Exp Brain Res 79:271-282

Durbin R, Mitchison G (1990) A dimension reduction framework for understanding cortical maps. Nature 343:644 647

Edwards DP, Kaplan E (1992) How sharp is the orientation tuning of single cells in the cytochrome-oxidase blobs of primate visual cortex (abstract). Invest Ophthalmol 33:1255

Elsdale R, Wasoff F (1976) Fibroblast cultures and dermatoglyphics: the topology of two planar patterns. Wilhelm Roux Arch Entwicklungsmech Org 180:121-147

Ferster D (1987) Origin of orientation-selective EPSPs in simple cells of cat visual cortex. J Neurosci 7:1780-1791

Frostig RD, Lieke EE, Ts'o DY, Grinvald A (1990) Cortical functional architecture and local coupling between neuronal activity and the microcirculation revealed by in vivo high-resolution optical imaging of intrinsic signals. Proc Natl Acad Sci USA 87:6082-6086

Götz G (1987) Do "d-Blob" and "l-Blob" hypercolumns tessellate the monkey visual cortex'? Biol Cybern 56:107-109

Götz. G (1988) Cortical templates for the self-organization of orientation-specific $\mathrm{d}$ - and l-hypercolumns in monkeys and cats. Biol Cybern 58:213-223

Grinvald A, Lieke E, Frostig RD, Gilbert CD, Wiesel TN (1986) Functional architecture of cortex revealed by optical imaging of intrinsic signals. Nature 324:361 -364

Hata Y, Tsumoto T, Sato H, Hagihara K, Tamura H (1988) Inhibition contributes to orientation selectivity in visual cortex of cat. Nature 335:815-817

Hess R, Negishi K, Creutzfeldt O (1975) The horizontal spread of intracortical inhibition in the visual cortex. Exp Brain Res 22:415-419

Hubel DH, Wiesel TN (1962) Receptive fields binocular interaction and functional architecture in the cat's visual cortex. J Physiol (Lond) 160:106 154

Hubel DH, Wiesel TN (1963) Shape and arrangement of columns in the cat's striate cortex. J Physiol (Lond) 165:559.568

Hubel DH, Wicsel TN (1968) Receptive fields and functional architecture of monkey striate cortex. J Physiol (Lond) 195:215-243

Hubel DH, Wiesel TN (1974) Sequence regularity and geometry of orientation columns in the monkey striate cortex. J Comp Neurol 158:267-243

Hubel DH, Wiesel TN, Stryker MP (1978) Anatomical demonstration of orientation columns in macaque monkey. J Comp Neurol $177: 361-380$

Humphrey AL. Norton TT (1980) Topographic organization of the orientation column system in the striate cortex of the tree shrew (Tupaia glis) I. Microelectrode recording. J Comp Neurol 192:531:547

Kohonen T (1990) The self-organizing map. Proc IFEE 78:1464 1480

Lee BB, Albus K, Heggelund P, Hulme MJ, Creutzfeldt OD (1977) The depth distribution of optimal stimulus orientations for neurones in cat area 17. Exp Brain Res 27:301-314

Legendy CR (1978) Cortical columns and the tendency of neighboring neurons to act similarly. Brain Res 158:89 105

Linsker R (1986a) From basic network principles to neural architecture: emergence of orientation-selective cells. Proc Natl Acad Sci USA 83:8390-8394

Linsker R (1986b) From basic network principles to neural architecture: Emergence of orientation columns. Proc Natl Acad Sci USA 83:8779-8783

Malsburg C von der, (1973) Self-organization of orientation sensitive cells in the striate cortex. Kybernetik: 14:85-100 
Malsburg C von der, Cowan JD (1982) Outline of a theory for the ontogenesis of iso-orientation domains in visual cortex. Biol Cybern 45:49 56

Miller KD (1990) Cortical organization of orientation selectivity emerges from interactions between $\mathrm{ON}$ - and OFF-center inputs. Soc Neurosci Abstr 16:798

Miller KD (1992) Development of orientation columns via competition between ON- and OFF-center inputs. NeuroReport 3:73-76

Nass MM, Cooper LN (1975) A theory for the development of feature detecting cells in visual cortex. Biol Cybern 19:1 18

Niebur E, Wörgötter F (1990) Circular inhibition: a new concept in long-range interactions in the mammalian visual cortex. In: Proceedings of the International Joint Conference on Neural Networks - San Diego '90. IEEE, Piscataway, New Jersey, pp II367-II-372

Obermayer K, Ritter H, Schulten K (1990) A principle for the formation of the spatial structure of cortical feature maps. Proc Natl Acad Sci USA 87:8345-8.349

Obermayer K, Blasdel GG, Schulten K (1991) A neural network model for the formation and for the spatial structure of retinotopic maps orientation- and ocular dominance columns. In: Kohonen $\mathrm{T}$ (ed) Artificial neural networks 1. North-Holland, Amsterdam, pp 505511

Orban GA (1984) Neuronal operations in the visual cortex. (Studies of brain function 11) Springer, Berlin Heidelberg New York

Perez R, Glass L, Shlaer R (1975) Development of specificity in the cat visual cortex. J Math Biol 1:275-288

Rojer AS, Schwartz EL (1990) Cat and monkey cortical columnar patterns modeled by bandpass-filtered $2 \mathrm{D}$ white noise. Biol Cybern 62:381-391

Soodak RE (1987) The retinal ganglion cell mosaic defines orientation columns in striate cortex. Proc Natl Acad Sci USA $84: 3936-3940$
Swindale NV (1982) A model for the formation of orientation columns. Proc R. Soc London 215:211 230

Swindale NV (1985) Iso-orientation domains and their relationship with cytochrome oxidase patches. In: Rose D, Dobson VG (eds) Models of the visual cortex. Wiley, New York, pp 452472

Swindale NV (1992) A model for the coordinated development of columnar systems in primate striate cortex. Biol Cybern 66:217 230

Swindale NV, Matsubara JA. Cynader MS (1987) Surface organization of orientation and direction selectivity in cat area 18 . J Neurosci $7: 1414 \quad 1427$

S7entagothai J (1975) The module concept in cerebral cortex architecture. Brain Res 95:475-496

Toyama K, Kimura M, Tanaka K (1981a) Cross-correlation analysis of interncuronal connectivity in cat visual cortex. J Neurophysiol 46: $191-201$

Toyama K, Kimura M, Tanaka K (1981b) Organization of cat visual cortex as investigated by cross-correlation technique. J Neurophysiol 46:202-214

Ts'o DY, Frostig RD, Lieke EE, Grinvald A (1990) Functional organization of primate visual cortex revealed by high resolution optical imaging. Science 249:333-348

Wörgötter F, Eysel UT (1991) Topographical aspects of excitatory and inhibitory intracortical interactions contributing to orientation specificity in cat visual cortex. Eur J Neurosci 3:1232-1244

Wörgötter F, Niebur E (1992) Generation of direction selectivity by isotropic intracortical connections. Neural Comp 4:332-340

Wörgötter F, Niebur E, Koch C (1990) Modeling visual cortex: hidden anisotropies in an isotropic inhibitory connection scheme. In: Eckmiller $\mathbf{R}$ (ed) Advanced neural computers. Elsevier, Amsterdam, pp 8795

Wörgötter F, Niebur E, Koch C (1991) Isotropic connections gencrate functional asymmetrical behavior in visual cortical cells. J Neurophysiol 66:444 459 